\title{
Dynamics of intermediate filament assembly followed in micro-flow by small angle $\mathrm{X}$-ray scattering
}

\author{
Martha Elisabeth Brennich, ${ }^{a}$ Jens-Friedrich Nolting, ${ }^{a}$ Christian Dammann, ${ }^{a}$ Bernd Nöding, ${ }^{a}$ Susanne Bauch, ${ }^{a}$ \\ Harald Herrmann, ${ }^{b}$ Thomas Pfohl ${ }^{c}$ and Sarah Köster ${ }^{* a}$
}

Received 20th August 2010, Accepted 14th December 2010
DOI: 10.1039/c0lc00319k

The assembly of intermediate filaments (IFs) is a complex process that can be recapitulated through a series of distinct steps in vitro. The combination of microfluidics and small angle X-ray scattering (SAXS) provides a powerful tool to investigate the kinetics of this process on the relevant timescales. Microfluidic mixers based on the principle of hydrodynamic focusing allow for precise control of the mixing of proteins and smaller reagents like ions. Here, we present a multi-layer device that prevents proteins from adsorbing to the channel walls by engulfing the protein jet with a fluid layer of buffer. To ensure compatibility with SAXS, the device is fabricated from UV-curable adhesive (NOA 81). To demonstrate the successful prevention of contact between the protein jet and the channel walls we measure the distribution of a fluorescent dye in the device by confocal microscopy at various flow speeds and compare the results to finite element method (FEM) simulations. The prevention of contact enables the investigation of the assembly of IFs in flow by gradually increasing the salt concentration in the protein jet. The diffusion of salt into the jet can be determined by FEM simulations. SAXS data are collected at different positions in the jet, corresponding to different salt concentrations, and they reveal distinct differences between the earliest assembly states. We find that the mean square radius of gyration perpendicular to the filament axis increases from $13 \mathrm{~nm}^{2}$ to $58 \mathrm{~nm}^{2}$ upon assembly. Thereby we provide dynamic structural data of a complex assembly process that was amenable up to now only by microscopic techniques.

\section{Introduction}

Intermediate filaments (IFs) are built from extended coiled-coil proteins and are principal components of the cytoskeleton of metazoan cells. Together with microtubules and microfilaments they form the intracellular network of these cells. The highly dynamic structures of IFs are not only important for the structural and mechanical integrity of the cell, but are also involved in organization and signaling processes. ${ }^{1,2}$ The assembly process of IFs differs fundamentally from the polymerization of microfilaments and microtubules. While the latter filaments polymerize from globular subunits by polar association, ${ }^{3}$ the assembly process of IFs is assumed to consist of several hierarchical substeps. One representative of the IF family is vimentin. Vimentin occurs in cells of mesenchymal origin such as fibroblasts or blood

${ }^{a}$ Courant Research Centre "Nano-Spectroscopy and X-ray Imaging", Georg-August-Universität Göttingen, Friedrich-Hund-Platz 1, 37077 Göttingen, Germany. E-mail: sarah.koester@phys.uni-goettingen.de; Fax: +49 (0)551399430; Tel: +49 (0)551399429

${ }^{b}$ Division of Molecular Genetics, German Cancer Research Center (DKFZ), Im Neuenheimer Feld 580, 69120 Heidelberg, Germany

'Biophysikalische Chemie, Departement Chemie, Universität Basel, Klingelbergstrasse 80, 4056 Basel, Switzerland vessel endothelium cells. In vitro, its smallest stable soluble complex is a tetramer consisting of two coiled-coiled dimers (length $60 \mathrm{~nm}$, the diameter can be estimated from ref. 4 to about $5 \mathrm{~nm}$ ). After increasing the salt concentration, tetramers rapidly laterally assemble into uniform short mini-filaments, also called unit-length filaments (ULFs, length $60 \mathrm{~nm}$, diameter $17 \mathrm{~nm}$ ). In a second phase, ULFs align longitudinally into filaments which then laterally compact to a diameter of $10 \mathrm{~nm} .{ }^{5}$ The kinetics of this mechanism have been worked out by time-lapse transmission electron microscopy (TEM) and atomic force microscopy (AFM) experiments. ${ }^{5-9}$

Microfluidic laminar flow mixers provide a suitable method to investigate reaction kinetics on time scales of sub-seconds to hours. This mixing can be realized by hydrodynamically focusing a solution of slowly diffusive macromolecules by a second solution flowing in from two orthogonal channels. Strongly diffusive small molecules or ions can then diffuse into the resulting macromolecule jet and initiate the assembly process as illustrated in Fig. 1a. The small dimensions of such mixers ensure laminar flow and allow for precise control of concentrations and flow conditions. ${ }^{10-12}$

Combining these devices with X-ray scattering allows for the observation of reaction kinetics on a molecular level in solution. 
For such experiments, it is necessary to design microfluidic devices from materials with small cross-sections for X-ray absorption and scattering processes. ${ }^{13-15}$ The devices used for these studies are usually tailor-built to account for the experimental requirements, e.g. accessible scattering vectors or temporal resolution. A variety of biomolecular processes, like collagen assembly or DNA condensation, have been studied by X-ray diffraction. The channels for these devices are spark eroded in stainless steel or produced by soft lithography techniques that allow for more complex structures. The channels are covered by adhesive Kapton film. ${ }^{16-20}$ Pollack et al. investigated the dynamics of protein and RNA folding by SAXS $^{21,22}$ using channels etched in silicon wafers and topped by thin polydimethylsiloxane (PDMS) films. ${ }^{21}$ In these studies so-called rapid mixers have been employed where the device dimensions and flow rates are chosen such that a complete diffusive mixing of the solutions can be achieved in minimal time.

In many reactions of interest, like the formation of protein assemblies or networks, undesired adhesion of reaction products to the channel walls prevents the formation of a stable flow. Several solutions to this problem have been proposed: ${ }^{23,24}$ One recurring feature is the prevention of contact between the adhering protein and the channel walls, for example by enclosing the protein in drops. ${ }^{24}$ Acoustically leviated liquid drops have been used to investigate protein crystallization ${ }^{25}$ and agglomeration. ${ }^{26}$ Such an approach is advantageous when one is interested in small compartmentalized sample volumes without continuous flow. Another approach, that is well suited for weakly diffusive proteins, is a variation of the mixer described above. In this case the buffer solution from the side channels engulfs the flow of the protein solution and forms a layer between the protein and the channel walls. ${ }^{21-27}$

For these reasons we propose a variation of the above mentioned device class in which additional buffer layers prevent contact between the channel walls and the protein. The device is made of moldable, UV-curable adhesive instead of PDMS or silicon. ${ }^{28,29}$ A major advantage of this material is its weak small

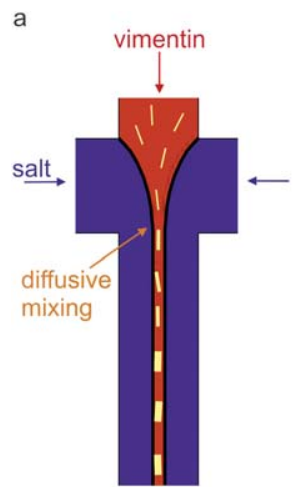

b

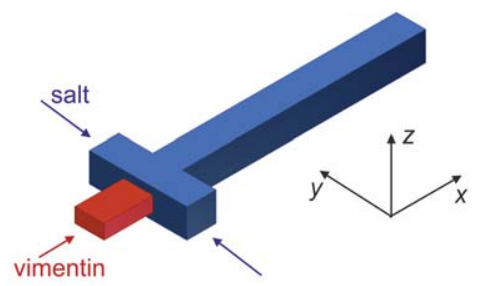

Fig. 1 Layout of a microfluidic laminar flow mixer. a) Schematic topview of the device. Vimentin solution enters the device from the central inlet, salt buffer from the sides. The buffer hydrodynamically focuses the vimentin solution to a jet. As it flows downstream, the salt diffuses into the vimentin jet and initiates the assembly process. b) Sketch of the microfluidic channel. The central inlet is shallower than the side inlets and the outlet, preventing contact between assembling vimentin and the channel walls. angle X-ray scattering which makes it suitable for SAXS studies. We investigate the influence of solute diffusion on the prevention of contact of the center stream and channel walls by measuring the distribution of a fluorescent dye in the device at varying flow speeds. Furthermore, we demonstrate that the device is suitable for the investigation of very fast assembling components such as vimentin. Finite element method (FEM) simulations allow us to predict the assembly conditions in the device. Combining these results we perform SAXS measurements at various positions in the device thereby probing the temporal development and concentration dependence of the assembly. We observe the lateral aggregation process by identifying three distinctly different scattering curves which can be identified as IF tetramers, sub-ULF intermediates and ULFs.

\section{Materials and methods}

\section{Photolithography}

Multi-layer photolithography of SU-8 negative resist (SU-8 3050, MicroChem, Newton, MA, USA) is used to fabricate masters for the device fabrication as shown in Fig. 2a-d. To produce even surfaces, the photolithography is performed in a class 100 cleanroom. In total, two layers of resist, 65 and $55 \mu \mathrm{m}$ thick, respectively, are spincoated onto the silicon wafer at 1800 $\mathrm{rpm}$. In order to prevent dewetting effects during the soft-baking process, a slight modification of the standard technique is used: a

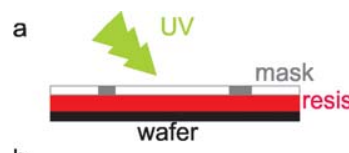

b

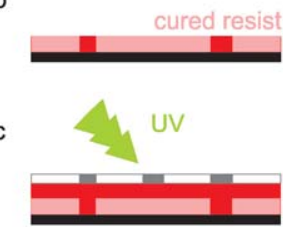

d
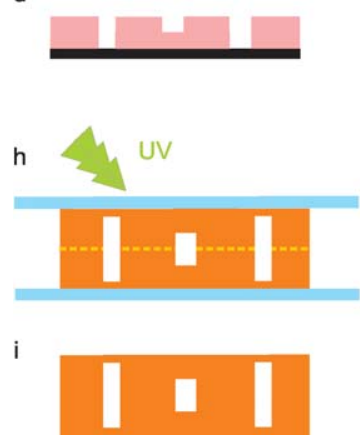
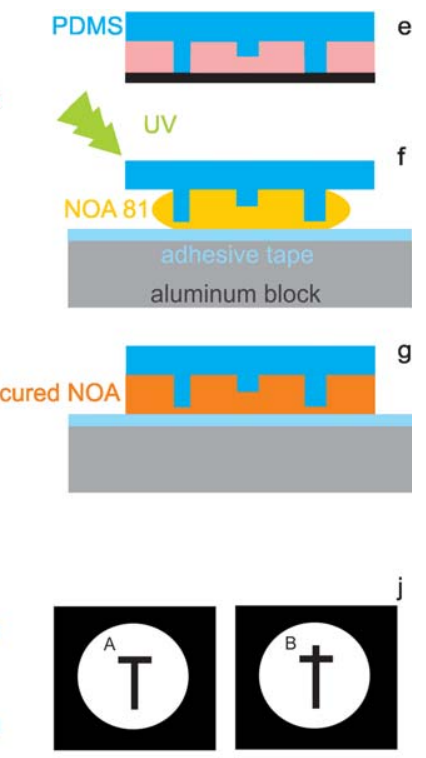

Fig. 2 Schematic overview of device construction: a)-d) Two layers of photoresist are consecutively spincoated on a wafer and exposed to UVlight. After the second exposure, the resist is developed and previously non-exposed regions become channels in the resist. e) A PDMS-stamp is cast from the wafer. f), g) The stamp is placed on a drop of NOA 81 which is then UV-cured. h), i) Two films of NOA 81 are aligned and cured together. j) Schematic of the photomasks used in steps a) and c). The letters $\mathrm{A}$ and $\mathrm{B}$ indicate the two different masks used in the two-layer lithography process. 
After applying the first layer, the temperature is slowly ramped (2 $\mathrm{K} \min ^{-1}$ ) to the final baking temperature of $90{ }^{\circ} \mathrm{C}$. This ramp is even slower for the second layer $\left(1 \mathrm{~K} \mathrm{~min}^{-1}\right)$, and the final baking temperature is only $80^{\circ} \mathrm{C}$. The first layer is exposed to UV-light through photomask A, the second layer through photomask B (Fig. 2j; JD Photo-Tools, Oldham, UK). Mask B is aligned to the existing structure on the wafer using a mask aligner (MJB4, SÜSS MicroTec, Garching, Germany). Between the application of the two layers, no development process takes place. After the second exposure the resist is developed. The resulting master is coated with (heptafluoropropyl)-trimethylsilane (Aldrich, Steinheim, Germany).

\section{Molding of UV-curable adhesive}

PDMS and crosslinker (Sylgard 184, Dow Corning, Midland, MI) are mixed at a ratio of $8: 1$ to create firm yet flexible stamps. The mixture is poured on a wafer produced by photolithography as described above and baked for $2 \mathrm{~h}$ at $65^{\circ} \mathrm{C}$ (Fig. 2e). $100 \mu \mathrm{L}$ of Norland Optical Adhesive 81 (NOA 81, Norland, Cranberry, NJ, USA) are placed on the structure on the stamp. Air bubbles are carefully removed with a thin pipette tip. Subsequently, another $180 \mu \mathrm{L}$ of NOA 81 are applied on a piece of adhesive tape (tesa Pack, tesa SE, Hamburg, Germany) attached to an aluminium block used as solid support. The transparent adhesive tape is a good base for the alignment process and can be easily removed after the final curing of NOA 81 . Then, the stamp is placed on the adhesive tape as shown in Fig. 2f. When the spreading NOA 81 film completely covers the structure, it is exposed to UV-light ( $8 \mathrm{~W}$ at $365 \mathrm{~nm}, 1 \mathrm{~min}$ ) (Fig. 2g). The exposure cures the NOA 81 except for a thin film at the NOA 81/ PDMS interface because PDMS facilitates the delivery of oxygen to the adhesive surface thereby inhibiting the curing. ${ }^{30,31}$ The PDMS stamp is removed and the NOA 81 film remains on the adhesive tape. A second NOA 81 film is produced in exactly the same way. Both films are placed atop of each other, with the structures facing each other (Fig. 2h). Further exposure to UVlight ( $8 \mathrm{~W}$ at $365 \mathrm{~nm}, 10 \mathrm{~min}$ ) cures them together (Fig. 2i). The resulting films are about $500 \mu \mathrm{m}$ thick. The buffer inlet channels and the outlet channel are $240 \mu \mathrm{m}$ high, the central inlet is 110 $\mu \mathrm{m}$ high. All channels are $300 \mu \mathrm{m}$ wide. Downstream of the intersection the outlet channel is $2 \mathrm{~cm}$ long. The main flow direction is the $x$-direction, $z$ is the height of the channel and $y$ the width. The origin $x=y=z=0$ is located in the center of the main channel at the position of the "step".

\section{Connection to the syringe system}

The NOA 81 microchannels need to be connected to the syringe system. Therefore, a stable connection with little dead-volume is required. We connect our devices via a flexible PDMS layer as follows: PDMS and crosslinker (Sylgard, Dow Corning) are mixed at a ratio of $10: 1$ and cast into disks of $5 \mathrm{~cm}$ diameter and $4 \mathrm{~mm}$ height $\left(2 \mathrm{~h}\right.$ baking time, $65^{\circ} \mathrm{C}$ ). The PDMS is glued to the structured NOA 81 film with Loctite Polyolefin (Loctite 406 and 770, Henkel, Düsseldorf, Germany), leaving measurement windows at the position of the structured regions of interest in the device. Before the Loctite completely hardens, holes for the inlets and outlets are punched through the NOA 81 film and the
PDMS. The holes on the NOA 81 side are closed with Kapton tape (Dr D. Müller GmbH, Ahlhorn, Germany) which provides a reliable, tight closure. On the PDMS side polyethylene tubing is placed into the holes and connected to the syringes (Hamilton Gastight, Hamilton Company, Reno, Nevada, USA). The syringes are operated by a remote-controllable pumping system (Nemesys, Cetoni, Korbußen, Germany).

\section{Protein purification and assembly}

Human vimentin protein is expressed in Escherichia coli (E. coli) bacteria and purified from inclusion bodies. ${ }^{32}$ The protein is stored at $-80{ }^{\circ} \mathrm{C}$ in $8 \mathrm{M}$ urea, $5 \mathrm{mM}$ Tris- $\mathrm{HCl}(\mathrm{pH} 7.5), 1 \mathrm{mM}$ EDTA, $0.1 \mathrm{mM}$ EGTA, $1 \mathrm{mM}$ DTT, and $10 \mathrm{mM}$ methyl ammonium chloride (MAC). The purity of the protein is verified by SDS-PAGE. Before use in the experiments, the protein is dialyzed into $4 \mathrm{mM}$ phosphate buffer (dialysis buffer) in a stepwise manner $(8,4,2,1,0 \mathrm{M}$ urea) using membranes of $50 \mathrm{kDa}$ cut-off. The protein concentration is determined by measuring the absorption at $280 \mathrm{~nm}$ (Nanodrop ND-1000, ThermoScientific Technologies, Inc.,Wilmington, USA). In vitro, assembly is initiated by addition of a salt buffer ( $4 \mathrm{mM}$ phosphate, $200 \mathrm{mM} \mathrm{KCl})$ to the vimentin solution at a $1: 1$ ratio. ${ }^{33} \mathrm{In}$ our experiment, vimentin $\left(4 \mathrm{mg} \mathrm{mL}^{-1}\right)$ is pumped into the central inlet at $335 \mu \mathrm{m} \mathrm{s}^{-1}\left(40 \mu \mathrm{L} \mathrm{h}^{-1}\right)$ and assembly buffer into the side inlets at $310 \mu \mathrm{m} \mathrm{s}^{-1}\left(80 \mu \mathrm{L} \mathrm{h}^{-1}\right)$.

\section{Simulation of the flow}

We use COMSOL multiphysics 4.0a (COMSOL GmbH, Göttingen, Germany) to perform FEM simulations of the flow conditions. The existence of two symmetry axes in the device allows us to reduce the computational effort by modeling only a quarter of the device (cut in the $\mathrm{x}-\mathrm{z}$ plane and the $\mathrm{y}-\mathrm{z}$ plane). To characterize the device we simulate the flow and the distribution of fluorescein in the device for various flow rates. The diffusion of fluorescein is modelled with the diffusion coefficient $D_{F}=4.25 \times 10^{-10} \mathrm{~m}^{2} \mathrm{~s}^{-1}$. The diffusion constant of tetrameric vimentin is estimated to be $D_{\text {vim }}=4.1 \times 10^{-11} \mathrm{~m}^{2} \mathrm{~s}^{-1} \cdot{ }^{34} \mathrm{We}$ neglect the decrease of the diffusion constant due to the filament growth during assembly to reduce the computational effort. The diffusion of $\mathrm{KCl}$ is modeled with $D_{K C l}=1.84 \times 10^{-9} \mathrm{~m}^{2} \mathrm{~s}^{-1}$.

\section{Microscopy}

DIC (differential interference contrast) microscopy images are obtained with a Leica TCS SP5 X confocal microscope with a $10 \times$ objective (Leica Microsystems, Wetzlar, Germany). Confocal fluorecence microscopy image stacks are recorded with an Olympus Fluoview 1000 CLSM equipped with a UPLSAPO $10 \times$ objective (Olympus, Tokyo, Japan). The vertical distance between single images is $4 \mu \mathrm{m}$. Controls for photo-bleaching reveal no bleaching effects. For the acquisition of intensity distributions at varying flow speeds, we started with experiments at high flow speeds gradually decreasing the speeds and equilibrating each flow rate. 


\section{X-ray scattering}

SAXS experiments are performed at the cSAXS (coherent small angle X-ray scattering) beamline at the Swiss Light Source, Paul Scherrer Institut, Villigen, Switzerland. The spot size is $5 \mu \mathrm{m} \times$ $20 \mu \mathrm{m}$. The photon energy is $11.2 \mathrm{keV}$ and the sample to detector distance $7.2 \mathrm{~m}$. Data are collected with a Pilatus 2M detector $\left(1475 \times 1679\right.$ pixels, pixel size: $172 \times 172 \mu \mathrm{m}^{2}$; SLS detector group, Villigen, Switzerland). The exposure time for each measurement is $5 \times 1 \mathrm{~s}$. The collected scattering images are radially integrated and corrected for contributions from buffer and device scattering. The assembly experiments are performed at room temperature.

In-house SAXS experiments for the device characterization are performed with a Bruker AXS Nanostar setup, including a rotating anode $\mathrm{X}$-ray source for $\mathrm{Cu}-\mathrm{K} \alpha$ radiation $(8 \mathrm{keV})$ at a generator power of $4.05 \mathrm{~kW}$ and a virtually noise-free, real-time 2D Hi-Star detector with photon counting ability (Bruker-AXS, Karlsruhe, Germany).

\section{Results and discussion}

\section{Device layout and construction}

We use a cross-shaped channel geometry as illustrated in Fig. 1b for our experiments. Tetrameric vimentin solution enters the device through the central inlet. At the cross intersection the vimentin solution is hydrodynamically focused by incoming high salt buffer from the side inlets. As the flow continues downward the main channel, the salt diffuses into the vimentin solution and starts the assembly process. To prevent the adsorption of assembled vimentin to the channel walls, we seek to cover the vimentin with salt buffer from all sides as soon as it enters the intersection. To achieve this, the vimentin inlet is about half as high as the buffer inlets and the downward channel.

The devices consist of two layers of photo-curable adhesive (NOA 81) each of which contain an open half of the channel and which are fused together to form the complete channel (Fig. 2h,i). A schematic overview of the production process is shown in Fig. 2, for details of the various steps see Materials and Methods. A critical step in the device construction is the alignment of the two halves. The partial curing of the NOA 81 layers in our protocol simplifies the aligning process as compared to aligning of, e.g., PDMS layers. The transparency of the NOA 81 layers further facilitates the alignment. The layout of the channels is flexible, complex structures like meandering serpentines can be built. The connection of the tubing via PDMS is mechanically flexible, has little dead-volume and further stabilizes the device. The combination of master production by photolithography and channel production by soft lithography processes allows us to mass-produce the devices at low cost and with little restrictions on the device geometry.

\section{Device characterization}

In order to determine the suitability of NOA 81 for small angle X-ray scattering (SAXS) we performed in-house experiments of both NOA 81 films and devices filled with liquid crystal 4 '-octyl4-biphenylcarbonitrile (8CB, Sigma, Steinheim, Germany). 8CB shows a distinc peak at $q=1.98 \mathrm{~nm}^{-1}$ while the small angle signal is weak and is therefore well suited for these preliminary tests of materials. We find that the small angle scattering of X-rays in NOA 81 is very small in comparison to e.g. adhesive Kapton foil or PDMS, which are used in other types of X-ray compatible microfluidic devices (data not shown). These results together with the advantages of the production process and the geometric flexibility discussed above, render NOA 81 devices very suitable for time-resolved SAXS studies.

To characterize the flow and solute distribution in these devices we pump a $100 \mu \mathrm{M}$ fluorescein solution into the central inlet and water into the side inlets. We observe the resulting fluorescein concentration at various flow speeds between $193 \mu \mathrm{m} \mathrm{s}^{-1}\left(50 \mu \mathrm{L} \mathrm{h}^{-1}\right)$ and $25 \mathrm{~mm} \mathrm{~s}^{-1}\left(6500 \mu \mathrm{L} \mathrm{h}^{-1}\right)$ by confocal microscopy and compare the results to FEM simulations (Fig. 3). The variation of the flow speeds allows us to determine the effects of diffusion on the spatial distribution of the solute. As the distributions at higher flow speed correspond to those of proteins, we will focus on the results for these speeds. The simulations assist in the interpretation of the data and allow us to distinguish between convection and diffusion effects. We find that the fluorescein flow gets focused in the $x-y$ plane upon entry of the cross. At low flow speeds an immediate broadening of the flow due to diffusion is observed as shown in Fig. 3a. We further find that for all flow speeds the fluorescein distribution expands in $z$-direction perpendicular to the main flow direction directly after entering the intersection (Fig. 3b,c). For high flow speeds this expansion can be solely attributed on the turning of the streamlines as depicted in Fig. 3c. At lower flow speeds, the distribution is further
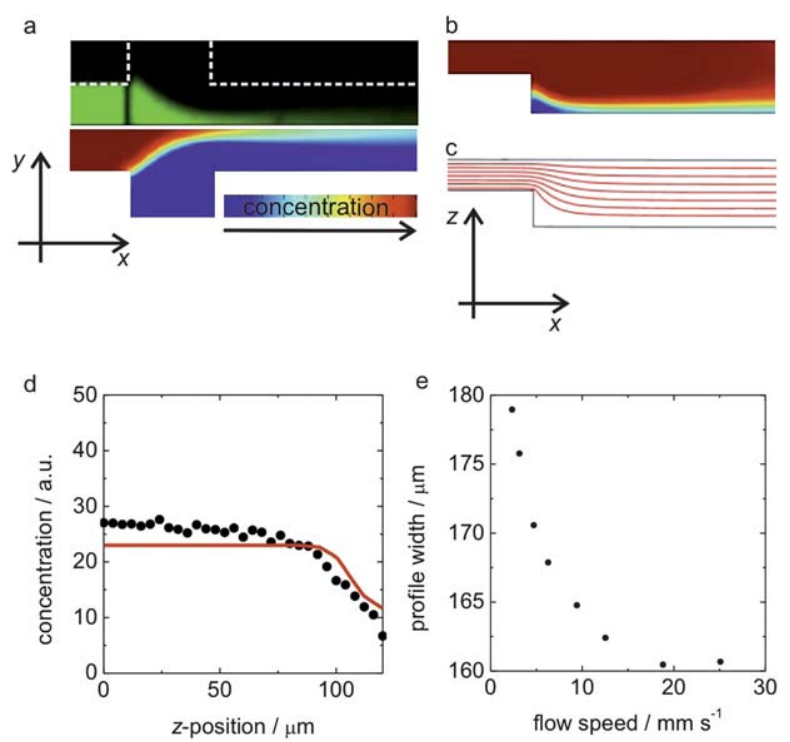

Fig. 3 Distribution of fluorescein in the device. a) Comparison of experiment and simulation, top-view: fluorescein distribution in the midplane of the device at $193 \mu \mathrm{m} \mathrm{s}^{-1}\left(50 \mu \mathrm{L} \mathrm{h}^{-1}\right)$ flow. The fluorescein diffuses towards the channel walls as it flows downwards. The channel walls are marked by dashed lines. b) Simulation of the fluorescein distribution, view on the central $x-z$ plane at $12.5 \mathrm{~mm} \mathrm{~s}^{-1}\left(3250 \mu \mathrm{L} \mathrm{h}^{-1}\right)$ flow. c) View on the central $x-z$ plane of the simulated streamlines at the intersection at $12.5 \mathrm{~mm} \mathrm{~s}^{-1}\left(3250 \mu \mathrm{L} \mathrm{h}^{-1}\right)$ flow. The streamlines from the central inlet turn downwards but do not reach the bottom of the channel. d) $z$-profile of the fluorescein distribution at the center of the intersection, at $12.5 \mathrm{~mm}$ $\mathrm{s}^{-1}\left(3250 \mu \mathrm{L} \mathrm{h}^{-1}\right)$ flow. Line: simulation, circles: experiment. e) The width of the $z$-profile of the fluorescein distribution $2 \mathrm{~cm}$ downstream of the intersection decreases with the flow speed. 
broadened by diffusion. At a high average flow speed of $12.5 \mathrm{~mm} \mathrm{~s}^{-1}$ $\left(3250 \mu \mathrm{L} \mathrm{h}^{-1}\right)$ the concentration profile along the $z$-axis in the middle of the intersection is relatively constant in the center of the jet and then rapidly vanishes (Fig. 3d). This corresponds well to the simulated concentration profile. Note that in the experiment the value does not decrease to zero due to the finite size of the confocal volume while in the simulation a similar effect is observed, which is due to the size of the finite elements. In general, the observed concentration distributions are in good agreement with the simulation. At sufficiently high flow speeds the fluorescein remains constricted to a thin sheet formed by the streamlines even further downstream as diffusion effects remain negligible. At lower flow speeds the fluorescein distribution in both the $y$-and $z$-direction broadens to eventually fill out the complete channel width and height (Fig. 3a). We observe that as we decrease the flow speed the $z$-profile becomes more stepwise and flat. This can be quantified by determining the width of the fluorescein distribution perpendicular to the flow at roughly $2 \mathrm{~cm}$ downstream from the intersection by calculating its standard deviation from the center (Fig. 3e). For the highest observed flow speeds, the distribution is relatively slim as there is little diffusion. As the flow becomes slower, the increasing influence of diffusion broadens the profile until it fills up the complete height of the device and fluorescein reaches the channel walls.

These results show that the ability of the device to prevent contact between the channel walls and the fluorescein depends on the speed of flow. In laminar mixers, the transport processes determining the solute distribution are convection along the streamlines and diffusion in all directions..$^{35-37}$ The characteristic dimensionless number for transport processes which relates the active transport of mass due to convection to the transport due to diffusion is the Péclet number

$$
P e=\frac{L v}{D} .
$$

Here $L$ is the typical length scale of the flow (e.g. the channel diameter), $v$ the velocity and $D$ the diffusion constant of the solute. At high flow speeds convection dominates and the solute remains restricted to the streamlines. At low flow speeds the transport is dominated by diffusion and the solute distributes through the whole device. Two transport processes A and B correspond to each other when they have the same Péclet number, i.e.

$$
P e_{A}=\frac{L_{A} v_{A}}{D_{A}}=P e_{B}=\frac{L_{B} v_{B}}{D_{B}},
$$

where the label A denotes the parameters of process $A$ and label $\mathrm{B}$ those of process $\mathrm{B}$. This allows us to draw conclusions on the distribution of vimentin in the device based on our results of the fluorescein distribution. Because we use the same device layout for both the fluorescein experiments and the vimentin assembly experiments their characteristic length scales are identical: $L_{F}=$ $L_{\text {vim }}$. Therefore, corresponding transport conditions exist when

$$
v_{F}=\frac{D_{F}}{D_{\text {vim }}} v_{\text {vim }}
$$

The diffusion constant of fluorescein $\left(D_{F}=4.25 \times 10^{-10} \mathrm{~m}^{2}\right.$ $\left.\mathrm{s}^{-1}\right)$ is about one order of magnitude higher than the diffusion constant of many proteins. Therefore, the distribution of fluorescein at the highest flow speeds described above (on the order of $1 \mathrm{~mm} \mathrm{~s}^{-1}\left(260 \mu \mathrm{L} \mathrm{h}^{-1}\right)$ to $10 \mathrm{~mm} \mathrm{~s}^{-1}\left(2600 \mu \mathrm{L} \mathrm{h}^{-1}\right)$ in the main channel) corresponds to the distribution of typical proteins at flow speeds on the order of $100 \mu \mathrm{m} \mathrm{s}^{-1}\left(26 \mu \mathrm{L} \mathrm{h}^{-1}\right)$ to $1 \mathrm{~mm} \mathrm{~s}^{-1}$ $\left(260 \mu \mathrm{L} \mathrm{h}^{-1}\right)$. We estimate the diffusion constant of tetrameric vimentin to be $D_{\text {vim }}=4.1 \times 10^{-11} \mathrm{~m}^{2} \mathrm{~s}^{-1}$ based on its molecular weight. ${ }^{34}$ This estimate for the diffusion constant does not account for the non-spherical shape of the assemblies. For diffusion perpendicular to the long axis of the cylinder-shaped tetramers, the value will be slightly decreased, while it increases (by a factor of 2 at most), when assuming diffusion in parallel to the long axis. Furthermore, the tetramer diffusion constant is the largest diffusion constant found in our system since larger assemblies (e.g. ULF) have smaller diffusion constants. This implies that for the typical flow speeds in the experiments described above, the distribution of vimentin corresponds to the distribution of fluorescein at flow speeds roughly one order of magnitude higher. For these high flow speeds, we find no contact between fluorescein and the channel walls besides the inlet channel. Therefore, at the flow speeds we use, the assembled vimentin can be safely assumed to have no contact to the channel walls. The stable flow conditions observed for the vimentin assembly further confirm the absence of vimentin/channel wall contact. These results show that we succeed in designing microfluidic devices which prevent the adsorption of protein to the channel walls. In combination with the above proof of suitability for SAXS experiments we therefore demonstrated that the devices allow for SAXS studies on assembly processes of biomolecules, like intermediate filament proteins.

\section{Vimentin assembly in the device}

For the investigation of the vimentin assembly a $4 \mathrm{mg} \mathrm{mL}-1$ solution of vimentin is pumped into the central inlet at $335 \mu \mathrm{m}$ $\mathrm{s}^{-1}\left(40 \mu \mathrm{L} \mathrm{h}^{-1}\right)$. From the side inlets, salt buffer $(200 \mathrm{mM} \mathrm{KCl})$ is pumped at twice of this flow rate each, resulting in about the same flow speed due to the increased channel height. The resulting average flow speed after the intersection is $770 \mu \mathrm{m} \mathrm{s}^{-1}$ $\left(200 \mu \mathrm{L} \mathrm{h}^{-1}\right.$ ). The total volume of buffer per unit time is exactly four times the volume of vimentin solution per unit time. This leads to a maximum concentration of $160 \mathrm{mM} \mathrm{KCl}$ in the vimentin jet. However, most of the data discussed here are taken at lower $\mathrm{KCl}$ concentrations, below $100 \mathrm{mM}$. We observe the resulting flow conditions by confocal DIC microscopy where the change in the optical density renders the edge of the vimentin flow visible. We find that the buffer smoothly focuses the vimentin solution from $300 \mu \mathrm{m}$ at the beginning of the intersection to roughly $75 \mu \mathrm{m}$ at the end of the intersection (Fig. 4a, upper part, edge indicated by dashed line for contrast enhancement). From the simulations (see below), we obtain values for the height (about $200 \mu \mathrm{m}$ ) and the width (about $60 \mu \mathrm{m}$ ) for the sheetlike jet in the center of the device. Consequently, as expected from the results of the fluorescein characterization, we observe no adsorption of protein to the channel walls, neither in DIC microscopy nor in SAXS experiments. By comparison, when using 2D flow-focusing devices adsorption and accumulation of protein at the channels walls can easily be detected using either method within a few minutes after the start of the flow 
experiment. In the 3D devices presented here the flow conditions are stable for several hours. In parallel, we image vimentin structures formed under identical buffer conditions by electron microscopy. After one second of assembly, a massive formation of ULFs of standard size ${ }^{6}$ is observed (data not shown).

\section{Simulation of vimentin flow}

As the assembly process of vimentin depends on both the exact salt and vimentin concentrations, ${ }^{33,38,39}$ a precise knowledge of their distributions in the device is necessary to further our understanding of the reaction kinetics. In order to access the distribution of both vimentin and salt in the device we perform FEM simulations, as we did for the fluorescein distribution for the device characterization. In these simulations we model a slight increase in viscosity as compared to water or buffer both for tetramer solution $\left(\eta_{\text {tet }}=1.2 \mathrm{mPas}\right)$ and upon assembly of the vimentin $\left(\eta_{\text {vim }}=2.8 \mathrm{mPas}\right)$, extrapolated from literature values. ${ }^{33}$ Diffusion of salt ions into the vimentin jet is not influenced by the presence of the protein assemblies, in accordance with previous work. ${ }^{17,19}$ It has been reported that after the addition of salt to the vimentin solution the viscosity increases and approaches a final value on the timescale of minutes. ${ }^{38,39}$ This time-dependence of the viscosity can either be due to the intrinsic reaction speed or diffusion limited. We assume that the assembly is diffusion limited in bulk and that the increase of the viscosity on the microfluidic length scale is likely to be instantaneous. Therefore, we only consider the final viscosities observed in these experiments. These final viscosities depend on both the vimentin concentration and the salt concentration.

According to the results of the simulations the buffer focuses the vimentin into a jet in the middle of the device. The vimentin concentration throughout the jet is nearly constant and the stream does not broaden due to diffusion (Fig. 4b, lower part). Due to the device design, the shear rates in the jet, which is located in the center of the fluid stream, are very low and have little or no influence on protein-protein interactions involved in the assembly process. The salt diffuses into this jet from the sides (Fig. 4b, upper part) and after $5 \mathrm{~mm}$, the salt concentration is homogeneous. We also find that there is a thin layer of buffer on the top and the bottom of the vimentin jet that prevents contact between the vimentin and the channel walls. Due to the thinness of these layers very little salt diffuses from them into the vimentin jet and the main contributions to the increase of the salt concentration in the jet can be attributed to diffusion from the sides (Fig. 4c).

To control for agreement of the simulation and the experiment we compare the simulated vimentin distribution to confocal DIC microscopy images of the assembly. We find that the edge of the flow observed in the DIC images corresponds well to the edge of the vimentin jet in the simulations (Fig. 4a). The good agreement justifies the approximations on the viscosity increase we employed in our model. The salt distribution in the device implies that by probing the vimentin jet perpendicular to the flow direction with an X-ray beam, mostly vimentin at one salt concentration contributes to the observed scattering signal (Fig. 4c).

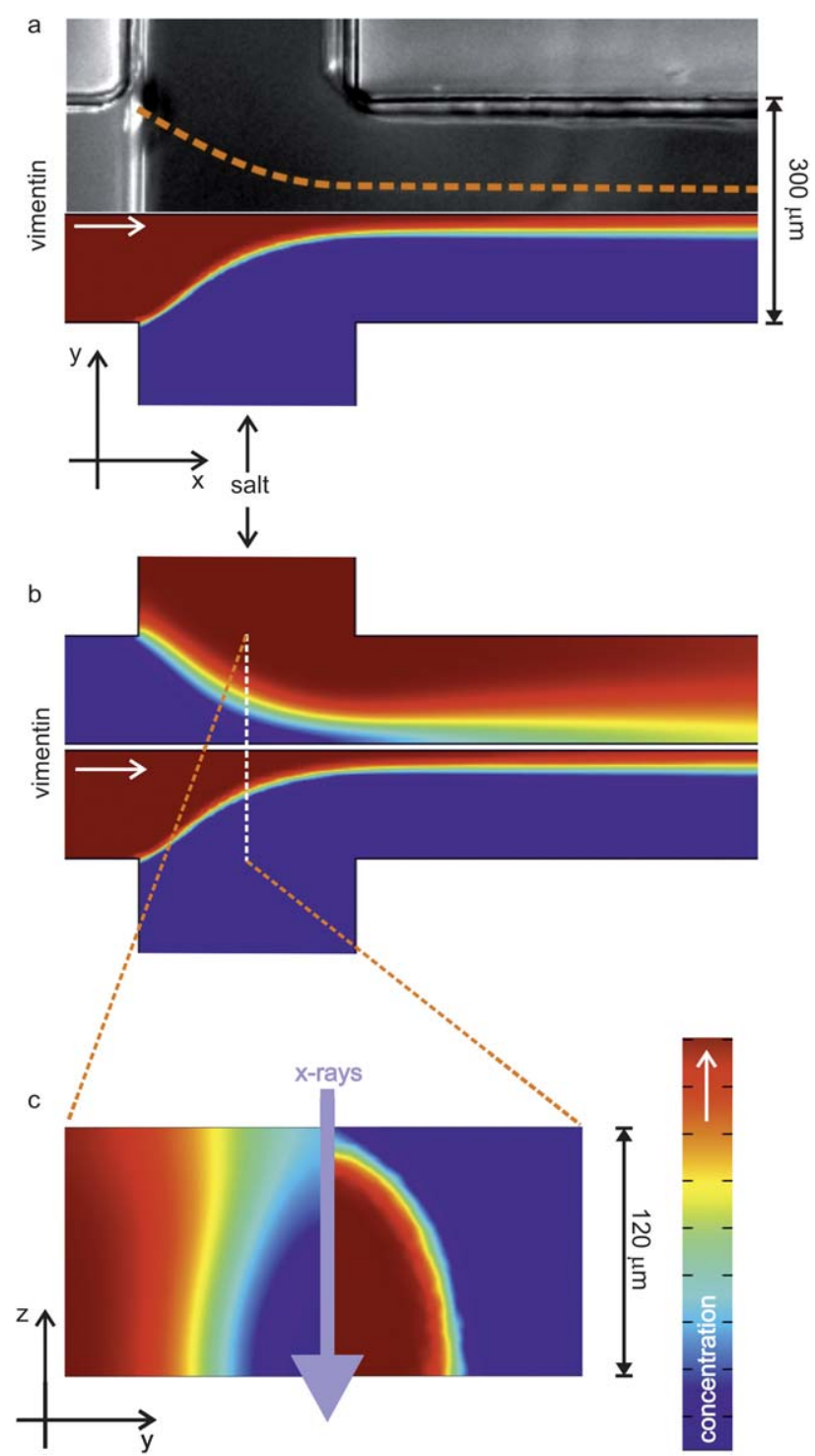

Fig. 4 Simulation of the vimentin and salt concentrations in the assembly process. The vimentin flow speed is $335 \mu \mathrm{m} \mathrm{s}^{-1}\left(40 \mu \mathrm{L} \mathrm{h}^{-1}\right)$ and the buffer flow speed at each inlet is $310 \mu \mathrm{m} \mathrm{s}^{-1}\left(80 \mu \mathrm{L} \mathrm{h}^{-1}\right)$. a) Mid-plane of device in the $x-y$ direction. The lower part of the image shows the simulation of the vimentin concentration, the upper part a corresponding DIC image. The dotted line marks the border line in the DIC image. The vimentin distributions match well. b) Comparison of the salt concentration distributions (upper part) and the vimentin concentration distribution (lower part) in the mid-plane of device in the $x-y$ direction. The vimentin forms a thin jet into which the salt diffuses. c) Comparison of the salt concentration distributions (left part) and the vimentin concentration distribution (right part) in the middle of the intersection in the $y-z$ direction. The vimentin has no contact to the channel walls. The salt diffuses into the vimentin mostly from the sides. The purple arrow indicates a possible position of the X-ray probe.

\section{Investigation of vimentin assembly by SAXS}

For the observation of the filament assembly we investigate the intermediates by spatially resolved SAXS. The size of the X-ray beam at the position of the device is small $(5 \mu \mathrm{m} \times 20 \mu \mathrm{m})$ in comparison to the device dimensions allowing us to probe the assembly at various positions in the flow. Because of the constant 
flow in the device probing at a position further downstream corresponds to probing a later assembly time. A further advantage of the constant flow is the continuous exchange of material that minimizes radiation damage. At the flow rates employed here, the residence time of a protein in the X-ray beam is about 25 $\mathrm{ms}$, a timescale much smaller than what is usually used in bulk Xray experiments. We find the scattering signal to be constant throughout the vimentin inlet. After entering the intersection the overall scattering signal immediately increases and continues to increase as the vimentin flows downstream. The scattering intensity $(I)$ at low scattering vectors $(q)$ becomes higher and the decrease at lower scattering vectors steeper (Fig. 5b). The sketch in Fig. 5a indicates the positions where the shown data are taken. Two of these scattering signals were recorded upstream of the intersection, three in the intersection and the remainder downstream from the intersection.

Additional changes in the form of the scattering curves can be accessed by a Kratky plot $\left(I \cdot q^{2} v s . q\right)$ in Fig. $5 c^{40,41}$ The initial scattering curve of the inflowing vimentin subunits, which we identify as tetramers increases to a broad peak at about $0.3 \mathrm{~nm}^{-1}$ and than slowly decreases to zero. Directly after entering the

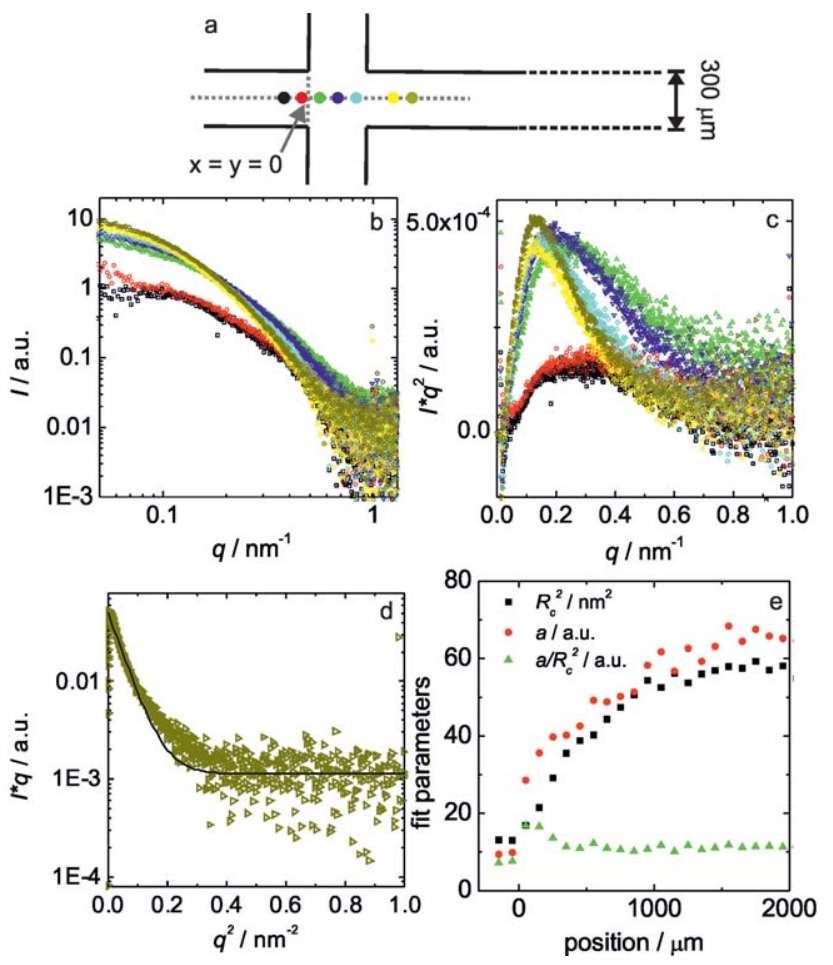

Fig. 5 Small angle X-ray scattering data from various positions in the flow. The positions of the illustrated data sets are depicted in the sketch of the cross. a) Sketch of the cross and measurement positions. The position $x=y=0$ is indicated by the gray arrow. The same color code is used in b)-d). b) Log-log plot of the scattering curves. After an initial increase the curves continue to become steeper at positions further down the flow. Such data sets have been taken for additional positions downwards the main flow channel (down to $x=2 \mathrm{~cm}$ ), however, changes become negligible after a certain distance. c) Kratky plot of the same data. Three different types of curves can be clearly distinguished. d) Example of a fit to a basic rod scattering model. The symbols indicate the original data, the black curve shows the corresponding fit. e) Dependence of fit parameters on the position, shown for $x$-positon values down to $2 \mathrm{~mm}$. intersection the increase in $I \cdot q^{2}$ at low scattering vectors becomes steeper whilst the decrease at higher $q$ values remains slow. The peak itself is shifted to lower scattering vectors $(0.25$ $\mathrm{nm}^{-1}$ ) and becomes higher. At positions further downstream the increase to lower scattering vectors continuous at a nearly constant peak height. As the peak moves to smaller scattering vectors it also narrows. About $1.25 \mathrm{~mm}$ after the beginning of the intersection no further significant changes in the Kratky plot are observed. We can distinguish three different types of Kratky plot curves: The flat and broad curves before assembly starts, the early taller but still broad curves and the final curves with narrow and distinct peaks. The existence of a peak implies that the observed states are mostly compact. For completely compact proteins the decrease in the Kratky plot follows $q^{-2}$ for high scattering vectors. ${ }^{42}$ We find a $q^{-1.3}$ dependence that hints at the existence of non-globular elements like Gaussian side-chains. The shift of the peak position indicates an increase of the radius and the increase in the peak height and increase in the over-all size or concentration.

To quantify these changes we apply a Guinier-like approximation for cylinder-like particles. ${ }^{42}$ In this approximation, the scattering curve is described by

$$
I(q) \cdot q=c L A \pi(\Delta \rho)^{2} e^{-q^{2} R_{c}^{2} / 2}
$$

where $L$ is the length of the cylinder, $A$ its cross-section, $R_{c}$ the radius of gyration of the cross-section, $c$ the concentration and $\Delta \rho$ the difference in electron density between solvent and solute. Note that the radius of gyration is defined as $R_{c}{ }^{2}=r^{2} / 2$ for solid cylinders with a radius of $r$ and as $R_{c}^{2}=r^{2}$ for hollow cylinders with a thin wall. Therefore, only in the latter case the value directly denotes the radius of the cylinder. For heterogeneous solutions the observed value of $R_{c}^{2}$ corresponds to the weighted mean of all contributors: $R_{c}^{2}=\sum_{i} f_{i} R_{c i}^{2}{ }^{43}$ As the vimentin tetramer complex is rather stiff and the presence of a peak in the Kratky plots indicates compactness this approximation is appropriate. By fitting the recorded data to this model we therefore obtain $R_{c}{ }^{2}$ and relative changes in $c L A$. An example fit of $I(q) \cdot q=a e^{-q^{2} R_{c}^{2} / 2}+d$ with the fit parameters $R_{c}^{2} / 2, a \propto c L A$ and $d$ is given in Fig. $5 \mathrm{~d}$ and the obtained fit parameters for the different positions are shown in Fig. 5e. We find that $R_{c}{ }^{2}$ starts to increase from $13 \mathrm{~nm}^{2}$ upon entry and eventually equilibrates at $58 \mathrm{~nm}^{2}$. The initial increase of the prefactor $a$ is much steeper, but after that it grows at about the same rate (Fig. 5e). Therefore the ratio of $a$ over $R_{c}^{2}$ remains constant except for the very beginning of the assembly.

Both the representation of the scattering data in the Kratky plot and the approximation of the scattering curves reveal a rapid increase in the radius of the macromolecular assemblies as one would expect for a lateral aggregation process. The final value for the square radius of gyration of the cross-section $\left(R_{c}^{2}=58 \mathrm{~nm}^{2}\right)$ is considerably larger than the value one would expect for mature, compacted filaments if they were perfect cylinders (12.5 $\mathrm{nm}^{2}$ ) or even for ULFs $\left(36.1 \mathrm{~nm}^{2}\right)$. However, neither mature filaments nor ULFs are perfect cylinders and especially ULFs are assumed to have a hollow ellipsoidal cross-section ${ }^{4}$ resulting in a considerably higher square radius of gyration $\left(72.3 \mathrm{~nm}^{2}\right.$ for a perfect ring) at equal radius. Our experimentally obtained value lies in-between the value for solid and hollow cylinders, as 
expected. Furthermore, we have to take into account that we most probably probe a heterogeneous mixture of different assembly states at a given position in the device rather than an absolutely homogeneous assembly state. Nevertheless the results confirm that at about $2 \mathrm{~mm}$ downstream of the cross-intersection mainly IF subunits which we can identify as ULFs are found. The initial increase in the ratio of $a$ over $R_{c}{ }^{2}$ is at least partially due to an increase of concentration as a consequence of the hydrodynamic focusing and the constant ratio thereafter indicates no observable increase in filament length.

The data shown here therefore allow us to access the dynamics of the lateral aggregation but not those of the subsequent elongation. By assigning each position to the flow time from the beginning of the intersection we can approximate a time constant for the aggregation in the device by fitting an exponential function to the increase of $R_{c}{ }^{2}$. We obtain a time constant of $0.75 \mathrm{~s}$ which is of the same order as the diffusion time of the salt into the vimentin jet.

\section{Conclusion}

Multi-layer microfluidic devices built of NOA 81 offer a very well-suited tool for the study of the reaction kinetics of biomolecular assembly processes by SAXS. The relatively slow diffusion of biomolecules enables these devices to prevent contact between the channel walls and the molecules and prevents adhesion. The design of our devices is flexible being based on lithographic methods. In combination with the variable flow rates, gradient mixers (as employed here) as well as fast mixers can be realized. The weak background SAXS signal of these devices allow for the detection of molecular scattering profiles at low additional noise. We apply these devices to the study of the assembly of intermediate filaments and can resolve sub-states of the lateral aggregation process. The correspondence of the aggregation time constant to the diffusion time of the salt indicates a diffusion-limited reaction. In future experiments, varying flow speeds and buffer salt concentrations will be used to further test these results. Furthermore, numerous additional applications to other biological or soft-matter systems are possible.

\section{Acknowledgements}

We thank Simon Castorph, Sravanti Uppaluri and Eric Stellamanns for fruitful discussions, Constantin Spille and Christian Westendorf for assistance with the confocal microscopy, Tatjana Wedig for help with electron microscopy, Jochen Herbst and Jörg Malindretos for technical support and Oliver Bunk for excellent support and discussions at the cSAXS beamline. We acknowledge the Swiss Light Source (SLS) for excellent working conditions and beam-time. This work was supported by the German Research Foundation (DFG) in the framework of SFB 755 "Nanoscale Photonic Imaging" and the Excellence Initiative and by the European Commission under the 7th Framework Programme: Research Infrastructures. Grant Agreement Number 226716.

\section{References}

1 J. E. Eriksson, T. Dechat, B. Grin, B. Helfand, M. Mendez, H. M. Pallari and R. D. Goldman, J. Clin. Invest., 2009, 119, 17631771 .
2 R. D. Goldman, B. Grin, M. G. Mendez and E. R. Kuczmarski, Curr. Opin. Cell Biol., 2008, 20, 28-34.

3 B. Alberts, A. Johnson, J. Lewis, M. Raff, K. Roberts and P. Walter, Molecular Biology of the Cell, Garland Science, 2007.

4 H. Herrmann and U. Aebi, Curr. Opin. Struct. Biol., 1998, 8, 177185.

5 R. Kirmse, S. Portet, N. Mücke, U. Aebi, H. Herrmann and J. Langowski, J. Biol. Chem., 2007, 282, 18563-18572.

6 H. Herrmann, M. Haner, M. Brettel, S. A. Muller, K. N. Goldie, B. Fedtke, A. Lustig, W. W. Franke and U. Aebi, J. Mol. Biol., 1996, 264, 933-953.

7 S. Portet, N. Mücke, R. Kirmse, J. Langowski, M. Beil and H. Herrmann, Langmuir, 2009, 25, 8817-8823.

8 L. Kreplak, K. Richter, U. Aebi and H. Herrmann, Introduction to Electron Microscopy for Biologists, Elsevier Academic Press Inc, San Diego, 2008, vol. 88, pp. 273-297.

9 A. V. Sokolova, L. Kreplak, T. Wedig, N. Mücke, D. I. Svergun, H. Herrmann, U. Aebi and S. V. Strelkov, Proc. Natl. Acad. Sci. U. S. A., 2006, 103, 16206-16211.

10 J. B. Knight, A. Vishwanath, J. P. Brody and R. H. Austin, Phys. Rev. Lett., 1998, 80, 3863-3866.

11 J. P. Brody, P. Yager, R. E. Goldstein and R. H. Austin, Biophys. J., 1996, 71, 3430-3441.

12 N. T. Nguyen and Z. G. Wu, J. Micromech. Microeng., 2005, 15, R1R16.

13 R. Barret, M. Faucon, J. Lopez, G. Cristobal, F. Destremaut, A. Dodge, P. Guillot, P. Laval, C. Masselon and J. Salmon, Lab Chip, 2006, 6, 494-499.

14 R. Dootz, H. Evans, S. Köster and T. Pfohl, Small, 2007, 3, 96-100.

15 L. Pollack, M. W. Tate, N. C. Darnton, J. B. Knight, S. M. Gruner, W. A. Eaton and R. H. Austin, Proc. Natl. Acad. Sci. U. S. A., 1999, 96, 10115-10117.

16 S. Köster, J. B. Leach, B. Struth, T. Pfohl and J. Y. Wong, Langmuir, 2007, 23, 357-359.

17 T. Pfohl, A. Otten, S. Köster, R. Dootz, B. Struth and H. M. Evans, Biomacromolecules, 2007, 8, 2167-2172.

18 R. Dootz, A. Otten, S. Köster, B. Struth and T. Pfohl, J. Phys.: Condens. Matter, 2006, 18, S639-S652.

19 S. Köster, H. M. Evans, J. Y. Wong and T. Pfohl, Biomacromolecules, 2008, 9, 199-207.

20 H. M. Evans, R. Dootz, S. Köster, B. Struth and T. Pfohl, Bull. Pol. Acad. Sci.: Tech. Sci., 2007, 55, 217-227.

21 R. Russell, I. S. Millett, M. W. Tate, L. W. Kwok, B. Nakatani, S. M. Gruner, S. G. J. Mochrie, V. Pande, S. Doniach, D. Herschlag and L. Pollack, Proc. Natl. Acad. Sci. U. S. A., 2002, 99, 4266-4271.

22 L. Pollack, M. W. Tate, A. C. Finnefrock, C. Kalidas, S. Trotter, N. C. Darnton, L. Lurio, R. H. Austin, C. A. Batt, S. M. Gruner and S. G. J. Mochrie, Phys. Rev. Lett., 2001, 86, 4962-4965.

23 W. Hellmich, J. Regtmeier, T. T. Duong, R. Ros, D. Anselmetti and A. Ros, Langmuir, 2005, 21, 7551-7557.

24 H. M. Evans, E. Surenjav, C. Priest, S. Herminghaus, R. Seemann and T. Pfohl, Lab Chip, 2009, 9, 1933-1941.

25 S. Santesson, E. Cedergren-Zeppezauer, T. Johansson, T. Laurell, J. Nilsson and S. Nilsson, Anal. Chem., 2003, 75, 1733-1740.

26 F. Delissen, J. Leiterer, R. Bienter, F. Emmerling and A. Thünemann, Anal. Bioanal. Chem., 2008, 392, 161-165.

27 M. Kinahan, E. Filippidi, S. Köster, H. Evans, T. Pfohl, D. Kaplan and J. Wong, unpublished work.

28 Z. T. Cygan, J. T. Cabral, K. L. Beers and E. J. Amis, Langmuir, 2005, 21, 3629-3634.

29 C. Harrison, J. T. Cabral, C. M. Stafford, A. Karim and E. J. Amis, J. Micromech. Microeng., 2004, 14, 153-158.

30 C. Decker and A. D. Jenkins, Macromolecules, 1985, 18, 1241-1244.

31 S. A. Stern, V. M. Shah and B. J. Hardy, J. Polym. Sci., Part B: Polym. Phys., 1987, 25, 1263-1298.

32 H. Herrmann, L. Kreplak and U. Aebi, Intermediate Filament Cytoskeleton, Elsevier Academic Press Inc, San Diego, 2004, vol. 78, pp. 3-24.

33 N. Mücke, T. Wedig, A. Burer, L. N. Marekov, P. M. Steinert, J. Langowski, U. Aebi and H. Herrmann, J. Mol. Biol., 2004, 340, 97-114.

34 M. E. Young, P. A. Carroad and R. L. Bell, Biotechnol. Bioeng., 1980, 22, 947-955.

35 D. A. Beard, J. Appl. Phys., 2001, 89, 4667-4669. 
36 R. F. Ismagilov, A. D. Stroock, P. J. A. Kenis, G. Whitesides and H. A. Stone, Appl. Phys. Lett., 2000, 76, 2376-2378.

37 A. E. Kamholz and P. Yager, Sens. Actuators, B, 2002, 82, 117121.

38 H. Herrmann, M. Haner, M. Brettel, N. O. Ku and U. Aebi, J. Mol Biol., 1999, 286, 1403-1420.

39 I. Hofmann, H. Herrmann and W. W. Franke, Cell Biol., 1991, 56, $328-341$.
40 M. Kojima, M. Tanokura, M. Maeda, K. Kimura, Y. Amemiya, H. Kihara and K. Takahashi, Biochemistry, 2000, 39, 1364-1372.

41 D. J. Segel, A. L. Fink, K. O. Hodgson and S. Doniach, Biochemistry, 1998, 37, 12443-12451.

42 O. Glatter and O. Kratky, Small Angle X-ray Scattering, Academic Press, London, 1982.

43 D. J. Segel, A. Bachmann, J. Hofrichter, K. O. Hodgson, S. Doniach and T. Kiefhaber, J. Mol. Biol., 1999, 288, 489-499. 